\title{
BMJ Open Cohort profile: the Comparative Outcomes And Service Utilization Trends (COAST) Study among people living with and without HIV in British Columbia, Canada
}

To cite: Eyawo 0, Hull MW, Salters K, et al. Cohort profile: the Comparative Outcomes And Service Utilization Trends (COAST) Study among people living with and without HIV in British Columbia, Canada. BMJ Open 2018:8:e019115. doi:10.1136/ bmjopen-2017-019115

- Prepublication history for this paper is available online. To view these files, please visit the journal online (http://dx.doi org/10.1136/bmjopen-2017019115).

Received 11 August 2017 Revised 2 November 2017 Accepted 22 November 2017

CrossMark

For numbered affiliations see end of article.

Correspondence to Dr Oghenowede Eyawo; oea1@sfu.ca

\section{ABSTRACT}

Purpose The Comparative Outcomes And Service Utilization Trends (COAST) Study in British Columbia (BC), Canada, was designed to evaluate the determinants of health outcomes and health care services use among people living with HIV (PLHIV) as they age in the period following the introduction of combination antiretroviral therapy (CART). The study also assesses how ageassociated comorbidities and health care use among PLHIV may differ from those observed in the general population.

Participants COAST was established through a data linkage between two provincial data sources: The BC Centre for Excellence in HIV/AIDS Drug Treatment Program, which centrally manages cART dispensation across BC and contains prospectively collected data on demographic, immunological, virological, cART use and other clinical information for all known PLHIV in BC; and Population Data $\mathrm{BC}$, a provincial data repository that holds individual eventlevel, longitudinal data for all 4.6 million BC residents. COAST participants include 13907 HIV-positive adults ( $\geq 19$ years of age) and a $10 \%$ random sample inclusive of 516340 adults from the general population followed from 1996 to 2013.

Findings to date For all participants, linked individuallevel data include information on demographics, health service use (eg, inpatient care, outpatient care and prescription medication dispensations), mortality, and HIV diagnostic and clinical data. Publications from COAST have demonstrated the significant mortality reductions and dramatic changes in the causes of death among PLHIV from 1996 to 2012, differences in the amount of time spent in a healthy state by HIV status, and high levels of injury and mood disorder diagnosis among PLHIV compared with the general population.

Future plans To capture the dynamic nature of population health parameters, regular data updates and a refresh of the data linkage are planned to occur every 2 years, providing the basis for planned analysis to examine ageassociated comorbidities and patterns of health service use over time.
Strengths and limitations of this study

A key strength of the COAST Study is its populationbased nature and the large sample size inclusive of all known HIV-positive adults in BC $(n=13907)$ and a $10 \%$ random sample of the general population of $B C$ as a comparison group $(n=516340)$.

- The COAST Study was based on an extensive data linkage between multiple population-level databases.

- COAST is situated within a universal health care setting, thus potential biases that typically result from differential access to health care services are minimised.

- Some of the data in COAST are based on administrative health data which can be prone to incomplete coding; therefore, common limitations associated with the use of such data for research remain.

\section{INTRODUCTION}

The introduction, widespread use and effectiveness of combination antiretroviral therapy (cART) since 1996 has led to marked improvements in the health of HIV-positive individuals and has contributed to a significant increase in life expectancy. ${ }^{1-4}$ As a result, HIV infection, which was once considered a fatal diagnosis, is now increasingly viewed as a chronic condition that can be managed effectively with lifelong use of $\mathrm{cART}^{5}{ }^{5}$ The population of ageing HIV-positive individuals is growing; in the USA, individuals over 50 years of age are estimated to make up over half of all people living with HIV (PLHIV). ${ }^{6}$ Ageing PLHIV may be at heightened risk for a number of chronic comorbid conditions, such as cardiovascular diseases, non-AIDS-defining cancers 
and other end-stage organ diseases. ${ }^{7-9}$ This increased risk has important implications in terms of managing HIV infection alongside other comorbid conditions. ${ }^{10}$

\section{Study rationale and aims}

Within the cART era-defined as starting in the year 1996 when cART became available-there is limited understanding regarding the impact of long-term cART use on ageing and age-related comorbidities among PLHIV. It is unclear how ageing with HIV infection will affect future patterns of morbidity and mortality among this population, and how this may consequently impact their patterns of health care use. For these reasons, we designed a longitudinal, population-based study-the Comparative Outcomes And Service Utilization Trends (COAST) Study-comprising a cohort of PLHIV and a comparative cohort based on a random sample of the general population of British Columbia (BC), Canada. The study aimed to: (1) evaluate the determinants or predictors of health outcomes and health care services use among HIV-positive men and women and how this relates to cART; (2) model the complex interaction between HIV and comorbidities to forecast the future health and economic impact of ageing with HIV; and (3) assess how health outcomes and health services use may differ between PLHIV and the general population from within the same health care system.

\section{COHORT DESCRIPTION}

\section{Study setting, data sources and available data}

The COAST Study is based on a unique data linkage performed between two provincial data sources in BC, namely, the BC Centre for Excellence in HIV/AIDS (BC-CfE) Drug Treatment Program (DTP) and Population Data BC. With a mandate from the government of BC, the BC-CfE's DTP centrally manages the distribution of cART to all PLHIV across BC. ${ }^{11}$ To monitor the trajectory of HIV disease and HIV-related outcomes, this programme prospectively collects data on cART use, demographic characteristics, immunological and virological outcomes, as well as other clinical characteristics (eg, hepatitis C co-infection and AIDS-defining illnesses) for all PLHIV accessing cART in BC. It is noteworthy to mention that all HIV care, including cART, laboratory and medical care, is provided free of charge to all PLHIV residing in BC. Population Data BC is a provincial repository that houses health administrative data collected by public bodies such as the BC Ministry of Health (BC-MoH), and contains an extensive range of individual-level, deidentified, longitudinal data for all 4.6 million $\mathrm{BC}$ residents. ${ }^{12}$

In $\mathrm{BC}$ as well as other provinces across Canada, each individual that uses any government-funded health care service is identified by a unique identifier, or the personal health number (PHN) as it is called in BC. PHN, which is available to all $\mathrm{BC}$ residents at birth or through immigration, is a unique lifetime identifier used to track individuals' encounters with the health care system and thus, their health care services use for the duration of residency in BC. Using unique identifiers such as PHN, name and date of birth, records for each individual are linkable across time and between internal data sets at Population Data BC, as well as to external data sets such as the BC-CfE DTP data that are held outside the Population Data BC data holdings.

For all participants in the COAST Study, individual-level data linkage to the following health administrative data sets held within Population Data BC was performed: (1) BC-MoH Medical Services Plan (MSP) (or the public health insurance programme), which insures medically necessary services provided by physicians and supplementary health care practitioners, and contains data on physician billing records; ${ }^{13}$ (2) BC-MoH Consolidation File for MSP registration and demographic data; ${ }^{14}$ (3) BC-MoH Discharge Abstract Database for hospitalisation records ${ }^{15}$ (4) Mental Health data set for mental health services and care information; ${ }^{16}$ (5) BC Cancer Agency registry for cancer-related diagnoses, treatment and care data; ${ }^{17}$ (6) BC Vital Statistics Deaths for mortality data; ${ }^{18}$ (7) PharmaCare for data on eligible prescription drugs and medical supplies covered under the provincial public drug insurance programme that assists $\mathrm{BC}$ residents with such payments; ${ }^{19}$ and (8) PharmaNet for all non-cART prescription drug dispensation, monitoring and claims processing data. ${ }^{20}$ Detailed information about these data sources is provided in table 1 . Based on the linkage, the available data in COAST include information on sociodemographic characteristics (eg, age, sex, neighbourhood information, health authority region); health care system encounters and other episodes of health care service use (eg, public health insurance registration data, inpatient and outpatient visits, and non-cART prescription medication dispensations including those covered by the $\mathrm{BC}$ public drug insurance programme (PharmaCare), as well as all dispensed medications in BC (PharmaNet)); mental health data; mortality data (centrally collected across BC); cancer-specific and HIV-specific data available through the BC Cancer Agency and BC-CfE (for PLHIV only), respectively. Within the COAST Study, data on diseases and other health outcomes such as injury can be assessed longitudinally using a combination of International Classification of Diseases (ICD) version 9 (ICD-9) and version 10 (ICD-10) diagnostic codes and prescription drug data (including the drug identification number, anatomical therapeutic chemical code), reflecting the specific health or disease state.

\section{COAST COHORTS}

Although the data used in creating the COAST Study (ie, data from the BC-CfE DTP and Population Data BC (including a collection of several data sources)) are collected longitudinally, the COAST Study follows a retrospective cohort study design. The follow-up period for the COAST Study is currently from 1 April 1996 (year of cART introduction) until 31 March 2013 (the 
Table 1 Description of data sources linked to create the COAST Study cohorts and key variables available

\begin{tabular}{ll}
\hline $\begin{array}{l}\text { Name of data } \\
\text { source* }\end{array}$ & $\begin{array}{l}\text { Data } \\
\text { stewardship }\end{array}$ \\
\hline $\begin{array}{l}\text { DTP and } \\
\text { laboratory } \\
11\end{array}$ & BC-CfE \\
& \\
MSP $^{13}$ & BC-MoH
\end{tabular}

\section{Description and content}

Summary: the BC-CfE DTP is a research and treatment programme that administers the distribution of CART in BC. The programme manages a database that collects information about cART use history, immunological and virological outcomes, laboratory testing results, and demographic information for HIV-positive individuals who have accessed cART in BC. Key data: CART use history, CD4 cell count, plasma viral load and demographic information.

BC-MoH Summary: the BC-MoH MSP Payment Information File contains data on medically necessary services provided by fee-for-service physicians, supplementary health care practitioners, laboratory services and diagnostic procedures (eg, X-rays, ultrasounds, etc), and dental and oral surgery for individuals covered by MSP, the BC province's universal health insurance programme.

Key data: laboratory and diagnostic procedures, encounter claims and all fee-for-service claims with dates, diagnoses and costs billed.

\begin{tabular}{|c|c|c|}
\hline $\begin{array}{l}\text { Consolidation } \\
\text { file }^{14}\end{array}$ & $\mathrm{BC}-\mathrm{MoH}$ & $\begin{array}{l}\text { Summary: this is the central demographics file used by Population Data } \mathrm{BC} \text { for research } \\
\text { requests. It pools data from multiple } \mathrm{BC}-\mathrm{MoH} \text { sources and includes information on individual } \\
\text { who receive health care services in } \mathrm{BC} \text {, and/or are registered and eligible to receive services } \\
\mathrm{BC} \text {. } \\
\text { Key data: basic demographics information such as age and sex, geographical codes } \\
\text { indicating location of residence. }\end{array}$ \\
\hline $\mathrm{DAD}^{15}$ & $\mathrm{BC}-\mathrm{MoH}$ & $\begin{array}{l}\text { Summary: the BC-MoH DAD captures demographic, administrative and clinical data for } \\
\text { hospital discharges (inpatient acute, chronic, rehabilitation) including transfers and deaths of } \\
\text { inpatients and day surgeries. } \\
\text { Key data: inpatient care data with information on care provided (eg, acute, day surgery, } \\
\text { rehabilitation, etc), diagnostic ICD-9 and ICD-10 codes in relation to patient's hospitalisation, } \\
\text { procedure and length of stay. }\end{array}$ \\
\hline
\end{tabular}

Mental health $\mathrm{BC}-\mathrm{MoH}$ services $^{16}$

\section{Cancer agency BC-CA} registry $^{17}$

$\begin{array}{ll}\begin{array}{ll}\text { Vital statistics } \\ \text { deaths }^{18}\end{array} & \begin{array}{l}\text { BC Vital } \\ \text { Statistics } \\ \text { Agency }\end{array} \\ \text { Pharmacare }^{19} & \text { BC-MoH }\end{array}$

$\begin{array}{ll}\text { Pharmanet }^{20} & \text { BC-MoH Data } \\ & \text { Stewardship } \\ & \text { Committee }\end{array}$

Summary: this data set contains information on care episodes and service events specific to mental health services.

Key data: mental health care episodes, service events and diagnosis assessed using DSM codes.

Summary: the BC-CA Registry contains information on all diagnosed and reported cancers for $\mathrm{BC}$ residents.

Key data: cancer diagnosis data including information such as demographics (age, sex) and other information such as diagnosis date, location of cancer, tumour group, histology.

Summary: this registry records information of all registered deaths in $\mathrm{BC}$.

Key data: includes information such as age and sex of deceased, date and place of death, and underlying cause of death coded using the ICD-9 and ICD-10 coding systems.

Summary: this data set captures information relating to prescription drugs covered under PharmaCare, the BC public drug insurance programme.

Key data: drug name/identifier including information such as prescriber code, prescription date and length, dosage, and drug cost.

Summary: this data set captures information relating to all prescription drugs dispensed by community and outpatient pharmacies in BC as well as PharmaCare claims.

Key data: drug name/identifier including information such as prescriber code, prescription date and length, dosage, and drug cost.

BC, British Columbia; BC-CA, BC Cancer Agency; BC-CfE, British Columbia Centre for Excellence in HIV/AIDS; BC-MoH, British Columbia Ministry of Health; cART, combination antiretroviral therapy; COAST, Comparative Outcomes And Service Utilization Trends; DAD, Discharge Abstract Database; DSM, Diagnostic and Statistical Manual of Mental Disorders; DTP, Drug Treatment Program; ICD, International Classification of Diseases; MSP, Medical Services Plan.

${ }^{*}$ Additional details describing these data sources can be found at: http://www.cfenet.ubc.ca and https://www.popdata.bc.ca/data.

period for which completely linked data are available). The study consists of two cohorts of adults ( $\geq 19$ years of age) (figure 1). The first, the HIV-positive cohort, is made up of all adults known to be HIV-positive between 1 April 1996 and 31 March 2013 in BC. The second, the general population comparison cohort, includes a $10 \%$ random sample of the total $\mathrm{BC}$ population meeting the age criterion ( $\geq 19$ years) between 1 April 1996 and 31 March 2013. Both cohorts are currently followed longitudinally from fiscal years 1996/1997 to 2012/2013 with future data updates planned biennially to enable us to capture the dynamic nature of population health parameters. Regular data updates and a refresh of the data linkage would allow us to extend the 


\section{COAST cohorts}

General population comparison cohort

Random $10 \%$ sample of distinct individuals aged 19+ in BC (1996-2013)

\section{HIV-positive cohort}

HIV-positive individuals aged $19+$ identified from databases and through HIV case-finding algorithm ${ }^{a, b}$

Temporal view of the COAST study

\section{COAST \\ Starts in \\ 1 April 1996 \\ Currently followed until 31 March 2013 \\ Future data updates planned biennially}

Figure 1 The COAST Study cohorts design and composition. BC, British Columbia; COAST, Comparative Outcomes And Service Trends. ${ }^{\mathrm{a}}$, Antoniou et al $2011^{14}$; b , Nosyk et al $2013 .{ }^{22}$

study follow-up to include more current years and additional data.

We defined loss to follow-up as no record of contact with the health care system as captured through an encounter or service event (eg, MSP registration and/or claim, hospitalisation or an HIV-related clinical test) within 18 months of the study cut-off date (currently 31 March 2013), in which case the individual's end of follow-up date corresponds to his/her last contact date. Deaths were not classified as loss to follow-up as all cohorts were linked to BC Vital Statistics Agency mortality data, ${ }^{18}$ containing information on all registered deaths in the province. In terms of the study's inclusion criteria, individuals become eligible for inclusion in the COAST Study if they reside in BC, have a PHN and are aged 19 years or older at either baseline or during the study follow-up. Details specific to the formation of the two COAST cohorts are described below.

\section{HIV-positive cohort creation}

We identified potential individuals for inclusion in the HIV-positive cohort through the BC-CfE DTP registry, with supplemental review of eight databases from Population Data BC (table 1). As a first step, we included all age-eligible individuals in the HIV-positive cohort if they had a record of at least one detectable HIV plasma viral load and/or have initiated cART (ie, individuals were contained in the BC-CfE DTP registry). In a second step, using ICD-9 and ICD-10 codes, we identified additional individuals for potential HIV-positive cohort inclusion by flagging those with at least one record of having received care for an HIV-related or AIDS-related medical condition or death through a review of supplementary administrative data sets in the databases described in table 1 . This case identification of potential HIV-positive individuals was enabled through the use of the following ICD codes: ICD-9 codes 042, 043, 044, V08, 795.71, 795.8; ICD-10 codes B20, B21, B22, B23, B24, R75, Z21; and ICD-10-CA codes B20-24, R75, Z21. These codes represent health states that are indicative of HIV infection. To distinguish true HIV-positive cases from those that may have been misclassified in the group of potential PLHIV identified through the administrative data sets, a validated HIV casefinding algorithm - an additional criterion of at least one inpatient and/or three or more outpatient ICD-9/10 codes associated with HIV/AIDS-was applied. ${ }^{21} 22$

\section{General population comparison cohort creation}

Within Population Data BC, the Consolidation File is the central demographics file and contains basic demographic information such as age, sex and geographical location of residence for all individuals who are eligible to or who receive health care services in $\mathrm{BC} .{ }^{14}$ We used this file to identify participants from the general population to serve as the comparison or control cohort. Specifically, to create the general population comparison cohort, a $10 \%$ random sample of persons from the demographics file was generated from a combined pool of distinct individuals with unique PHNs in the general population of BC meeting the age criterion ( $\geq 19$ years) from the fiscal years 1996/1997 to 2012/2013. A computer-generated simple random sampling technique was executed to draw the $10 \%$ random sample. A small number of HIV-positive individuals was randomly drawn and this allows us to further separate this cohort by serostatus and to perform analyses that compare PLHIV (ie, the HIV-positive cohort) to either the general population or to HIV-negative individuals, depending on the research question of interest. 


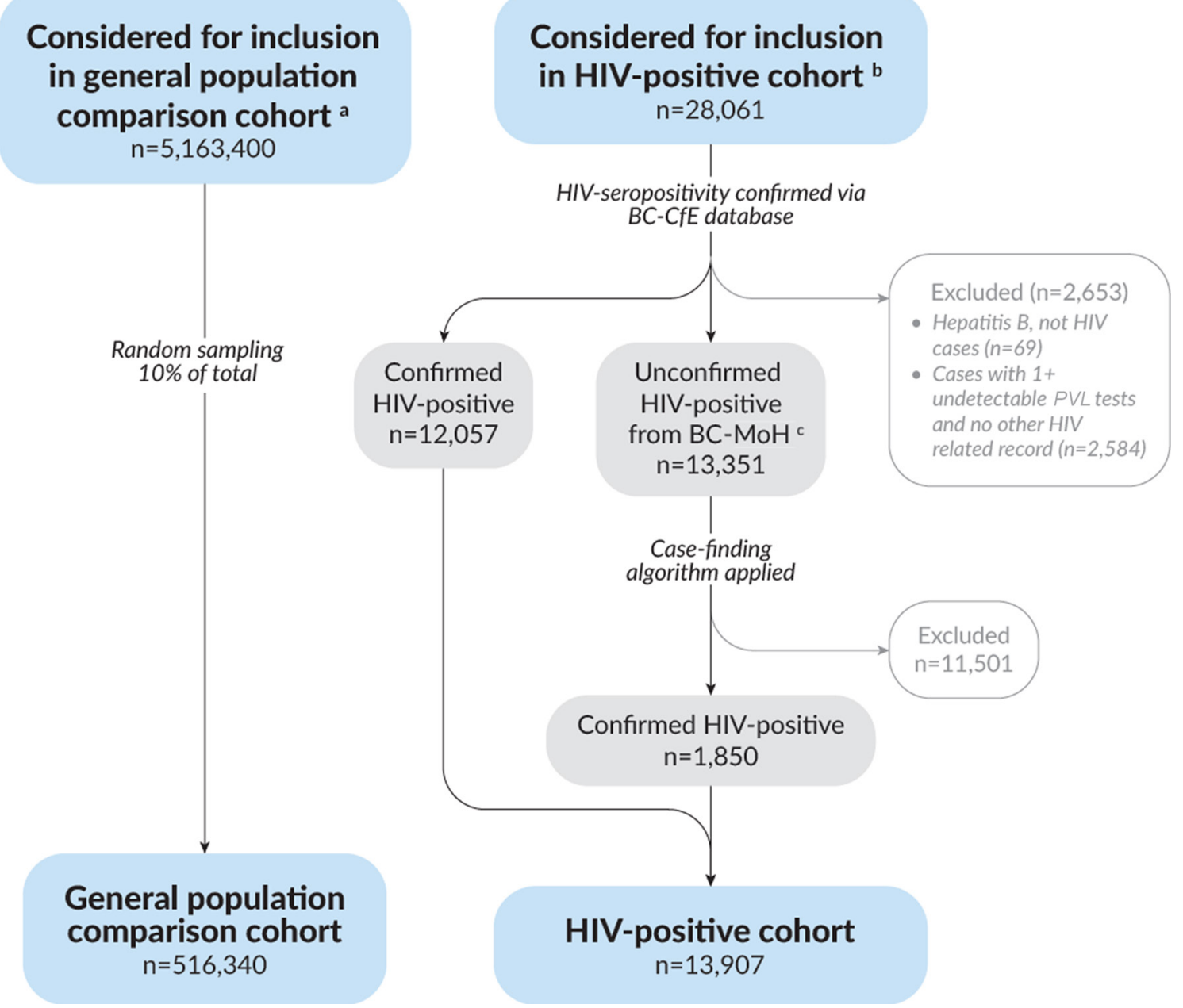

Figure 2 Flow diagram of the COAST Study cohorts creation process. ${ }^{a}$, represents all distinct individuals in BC who were 19 years or older between fiscal years 1996/1997 and 2012/2013; ${ }^{b}$, represents all individuals in BC who were 19 years or older between fiscal years 1996/1997 and 2012/2013 and with at least one record of the following: HIV-related hospitalisation or physician claim, AIDS-defining illness, antiretroviral therapy dispensation or a plasma viral load (pVL) test; ${ }^{c}$, represents unconfirmed HIV-positive cases with at least one HIV-related BC-MoH record, but no other HIV-related records in the BC-CfE database.

\section{Study participants and follow-up}

The COAST cohort creation process and the final composition of the two cohorts is described schematically in figure 2. In brief, the COAST Study, which is population-based in nature, includes 528859 unique individuals of whom 13907 (2.6\%) are HIV-positive. The median follow-up time (lower quartile (Q1), upper quartile $(\mathrm{Q} 3))$ is 7.1 years $(2.6,13.2)$ and 12.4 years (4.8, 16.9), for the HIV-positive and general population comparison cohorts, respectively. Table 2 provides a descriptive summary of the demographic composition and key characteristics of the study cohorts. The majority of individuals in the HIV-positive cohort were men $(80 \%)$ and the median age at entry into the study is 38 years $(32,46)$. About a quarter of the individuals in this cohort had no record of cART initiation during the current follow-up of this study. The general population comparison cohort includes 516340 individuals, $50 \%$ of whom were men. The median (Q1, Q3) age at baseline for this cohort is 35 years $(23,48)$.
Figures 3 and 4 graphically highlight the distribution of the study cohorts stratified by age and sex. As is shown in these population pyramids of individuals alive in 2013, there were considerably more men in the HIV-positive cohort than in the general population cohort. This population characteristic of the HIV-positive cohort is consistent with the demographic composition of the HIV epidemic in BC and across Canada. ${ }^{23}$ The male-to-female ratio in the general population comparison cohort was approximately 1 , but as expected, the proportion of men surviving to older ages ( $>70$ years) was relatively lower compared with women.

\section{Data linkages}

Data linkage allowed us to link records belonging to the same individual across multiple data sources and over time. In terms of the process, data linkages were performed by matching individuals in the two COAST cohorts to $\mathrm{BC}-\mathrm{MoH}$ registries and other administrative data sets through established linkage techniques 
Table 2 Characteristics of the COAST Study cohorts

\begin{tabular}{|c|c|c|c|}
\hline Variable & $\begin{array}{l}\text { HIV-positive cohort } \\
(n=13907)\end{array}$ & $\begin{array}{l}\text { General population } \\
\text { comparison } \\
\text { cohort }(n=516340)^{\star}\end{array}$ & $P$ value \\
\hline Age at baseline† median $(\mathrm{Q} 1, \mathrm{Q} 3)$ (years) & $38(32,46)$ & $35(23,48)$ & $<0.0001$ \\
\hline Sex, n (\%) & & & $<0.0001$ \\
\hline Male & $11161(80.2)$ & $259863(50.3)$ & \\
\hline Female & $2743(19.7)$ & $256144(49.6)$ & \\
\hline Unknown & $3(0.0)$ & $333(0.1)$ & \\
\hline Health authority, $\neq \mathrm{n}(\%)$ & & & $<0.0001$ \\
\hline Fraser & $2915(21.0)$ & $166803(32.3)$ & \\
\hline Interior & $913(6.6)$ & $83722(16.2)$ & \\
\hline Northern & $465(3.3)$ & $30973(6.0)$ & \\
\hline Vancouver Coastal & $7246(52.1)$ & $136747(26.5)$ & \\
\hline Vancouver Island & $1560(11.2)$ & $84650(16.4)$ & \\
\hline Unknown & $808(5.8)$ & $13445(2.6)$ & \\
\hline Follow-up time, median (Q1, Q3) years & $7.1(2.6,13.2)$ & $12.4(4.8,16.9)$ & $<0.0001$ \\
\hline Lost to follow-up,§ n (\%) & $1544(11.1)$ & $87004(16.9)$ & $<0.0001$ \\
\hline Alive as of 31 March 2013, n (\%) & $8858(63.7)$ & $380305(73.7)$ & $<0.0001$ \\
\hline \multicolumn{4}{|l|}{ Ever been on cART, $n(\%)$} \\
\hline Yes & $10210(73.4)$ & NA & NA \\
\hline No & 3697 (26.6) & & \\
\hline
\end{tabular}

cART, combination antiretroviral therapy; NA, not applicable; Q1, 25th percentile; Q3, 75th percentile.

*This cohort includes 1388 known HIV-positive individuals who were randomly drawn when the cohort was created. Depending on the research question, the HIV-positive individuals in this cohort can be excluded to have an HIV-negative cohort.

†Baseline is defined as the date of known positive HIV serostatus (if participant is HIV-positive) or the date when the age eligibility was met ( $\geq 19$ years of age), whichever came second, and a confirmed record of registration with the Medical Services Plan (MSP), the British Columbia (BC) public health insurance programme.

‡Refers to the provincial health care authority/jurisdiction where participants resided at latest known address.

$\S$ An individual is lost to follow-up when there has been no record of contact with the health care system as captured through an encounter or service event (eg, MSP registration and/or claim, hospitalisation, plasma viral load test, etc) for at least 18 months prior to the end of the study cut-off date (currently 31 March 2013), in which case the individual's end of follow-up date corresponds to his/her last contact date.

including manual, deterministic and probabilistic matching. Following linkage, the identifying data used for the linkage process were separated from the content data and the final deidentified data for research purposes were assigned with new non-identifying record IDs. Although the final linked data were deidentified and void of any identifying information, personal identifiers such as an individual's name, date of birth and unique PHN were used to facilitate the linkage process. The data linkage was executed by Population Data BC and approved by data stewards representing the various data providers, namely the BC-CfE, BC Cancer Agency, BC-MoH, BC Vital Statistics Agency and PharmaNet. Extensive detail on the data linkage process has been described by Population Data BC..$^{25}$

\section{Partner cohort studies}

COAST has two partner cohort studies with overlapping goals and objectives and coordinated within BC-CfE. These two studies (Highly Active Antiretroviral Therapy (HAART) Observational Medical Evaluation and Research (HOMER)) cohort, and the Seek and Treat for the Optimal Prevention of HIV/AIDS (STOP HIV/AIDS) cohort) which have been described extensively elsewhere, ${ }^{26}{ }^{27}$ use robust clinical data from the BC-CfE DTP registry to evaluate mortality, prognostic-related and treatment-related outcomes among HIV-positive individuals with the goal of informing treatment priorities and therapeutic guidelines among those living with HIV. Similar to COAST, the STOP HIV/AIDS Study also involves linkage to other provincial administrative databases to examine health care delivery indicators and evaluate the cascade of care and population-level effect of cART expansion. COAST is however more expansive in scope and has in addition to the HIV-positive cohort, a comparison cohort of over half million members of the general population. Furthermore, unlike HOMER that includes only cARTnaïve participants, ${ }^{26}$ and STOP HIV/AIDS that includes HIV-positive individuals aged 18 months and over, ${ }^{27}$ the COAST Study focuses primarily on HIV-positive adults irrespective of cART initiation, and the general population, to better understand health outcomes, health service use and long-term trends associated with HIV and ageing. 
Males: 7,098

Sex Ratio (m/f): 4.040

Females: 1,757

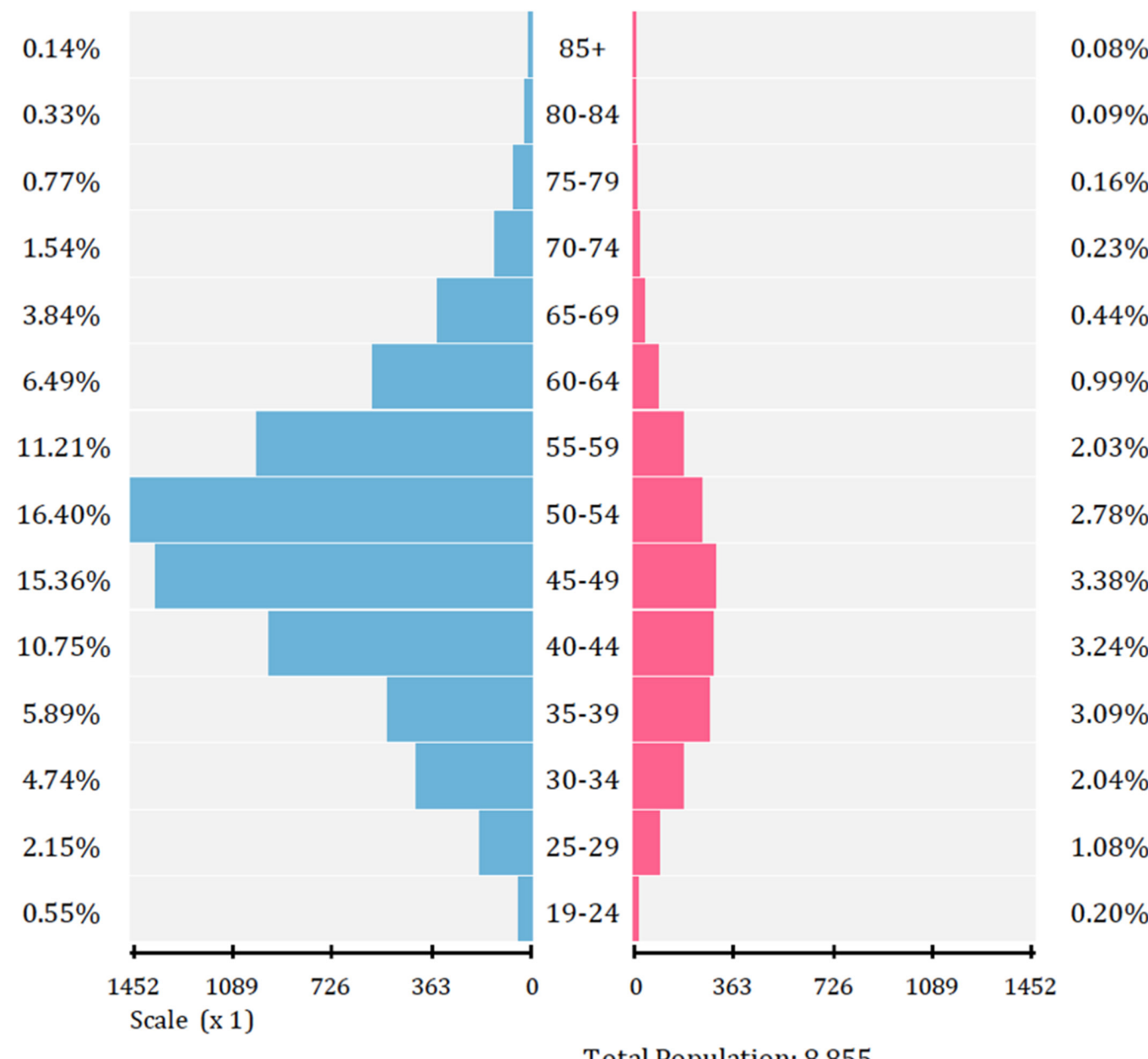

Total Population: 8,855

Figure 3 Population pyramid by age and sex for HIV-positive cohort members not lost to follow-up and alive as of 31 March 2013 (Note: those with unknown sex $(n=3)$ are not reflected in the figure).

\section{Findings to date}

In terms of research outputs, several investigations are currently underway or have already been completed since the data linkage and creation of the COAST Study in 2015. During the current follow-up from 1996 to 2013, a total of $3505(25 \%)$ and 49031 (9\%) individuals died in the HIV-positive and general population comparison cohorts, respectively. Regarding loss to follow-up, we observed the following: during the nearly 17 years of follow-up, approximately $11 \%$ and $17 \%$ of individuals have been lost to follow-up in the HIV-positive and general population comparison cohorts, respectively. These estimates are comparable to those reported in other major HIV cohorts. ${ }^{28}$ When we looked at the last 3 years of the study (1 April 2010 to 31 March 2013), approximately 3\% of participants were lost to follow-up in both groups.

The completed studies using the COAST data have been published in peer-reviewed journals and covered a range of health-related research topics with important clinical and policy relevance. One study examining health-adjusted life expectancy among adults with and without HIV sought to understand whether morbidity experienced by HIV-positive individuals on cART was compressed in the last years of life or lessened as people age. ${ }^{29}$ Results from this study found little differences in levels of morbidity compression by HIV status. However, PLHIV, notably women living with HIV, appeared to experience less time in a healthy state compared with those in the general population. Another study investigating the rates and predictors of injury within the COAST cohorts found that there were significantly higher rates of intentional and unintentional injury among HIV-positive individuals compared with the general population. ${ }^{30}$ We have also characterised the changing patterns in mortality rates and causes of death among HIV-positive and HIV-negative individuals in the period since the introduction of cART in $1996 .{ }^{31}$ This study found significant reductions in mortality among HIV-positive individuals in the period from 1996 to 2012. In particular, a reduction of over $90 \%$ in mortality rates from HIV-related causes was observed when rates in 1996 were compared with those in the 2011-2012 era. However, this study also pointed to the rise in mortality from comorbidities, such as non-AIDS-defining cancers, which were found to be 


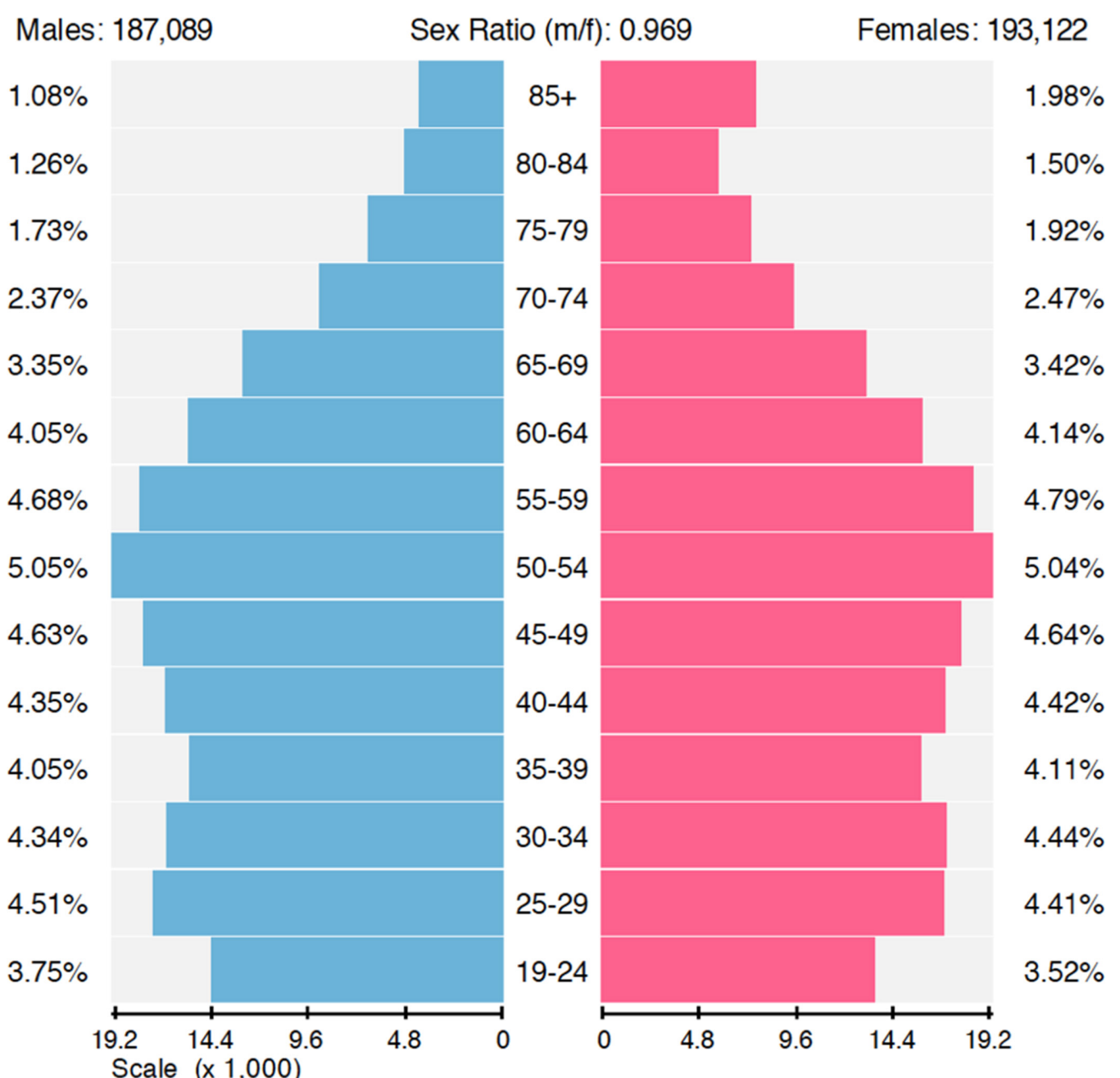

Total Population: 380,211

Figure 4 Population pyramid by age and sex for general population comparison cohort members not lost to follow-up and alive as of 31 March 2013 (Note: those with unknown sex ( $n=94)$ are not reflected in the figure).

currently the leading non-HIV-related cause of death in both HIV-positive and HIV-negative individuals. More recently, another publication by our group presented evidence demonstrating higher rates of mood disorder diagnosis among HIV-positive individuals compared with the general population. ${ }^{32}$ These publications using the COAST Study data, including several others in development, will provide additional understanding around these topics and contribute to important gaps in the literature.

\section{DISCUSSION}

The COAST Study is uniquely positioned to characterise, compare and forecast future health outcomes and health care service use in a population-based cohort of HIV-positive individuals as compared with those observed in the general population. Unlike other observational studies of HIV-positive individuals that lack a comparison group, we will be able to include additional data from the general population comparison cohort to document differences in ageing-related and disease-related outcomes between PLHIV and the general population over time. This will potentially help to identify and better examine key determinants that may help explain differences in health outcomes and trends in health care service use between PLHIV and the general population. Given that many comorbid conditions have overlapping aetiologies in both HIV-positive and HIV-negative individuals, ${ }^{33}$ this kind of comparison with the general population is becoming increasingly common in chronic disease studies, ${ }^{34-36}$ and can help identify morbidity and mortality gaps in PLHIV relative to the general population. Our hope is that this information will ultimately inform polices to guide treatment, management and service provision for ageing HIV-positive individuals in this province and elsewhere. Apart from the regular data updates and refresh to the linkage planned to occur every 2 years, future analysis will determine the validity of algorithms derived from administrative health data to identify chronic conditions and examine age-associated comorbidities and patterns of health service use over time among PLHIV and the general population. To date, the COAST Study has already established its value through 
key findings that demonstrate differences in health states according to HIV status, including highlighting the changes in the causes of death since cART's introduction among PLHIV. ${ }^{29-32}$

\section{Strengths and limitations}

There are several features of the COAST Study that reflect the study's strengths. First, the large sample size, population-based nature and representativeness of the study cohorts are all important elements of the study. In particular, the large sample size will allow us to assess small but potentially important differences in the outcomes of interest in the respective cohorts. Second, the HIV-positive cohort includes the vast majority of all known PLHIV in BC since our HIV case ascertainment method involved identification of clients from the BC-CfE registry combined with the use of a validated HIV case-finding algorithm to identify additional individuals from health administrative databases. The validated HIV case-finding algorithm helped reduce the risk of potential misclassification and increased our capacity to correctly identify true HIV-positive cases in these databases. ${ }^{22}$ Third, our use of a large comparison cohort of the general population comprised of individuals from within the same health care system as the HIV-positive cohort helps to minimise selection bias that can be introduced by using an external comparison group. The over half million unique participants that make up the general population comparison cohort were recruited across the time horizon of the study through a random selection process that implements the age eligibility criterion to ensure that the minimum age limit of this group is comparable to that of individuals in the HIV-positive cohort. Fourth, the cohorts within the COAST Study comprise individuals that access a publicly funded health care system-one that provides universal coverage for medically necessary health care services based on need rather than the ability to pay. Moreover, in BC, cART and related laboratory monitoring and medical care are fully subsidised, without any co-payments or deductibles, which are present in some other Canadian jurisdictions or elsewhere in the world. Therefore, potential biases that could result from differential access to health care services are minimised. Finally, the centralised nature of cART distribution in BC, which is managed by the BC-CfE DTP, ${ }^{11}$ is unique and has created an environment to facilitate population-based monitoring and evaluation of HIV-positive individuals' health outcomes and response to cART on a population-level basis. This feature of the BC-CfE DTP registry and the longitudinal follow-up of the study ensures additional opportunities for data capture and, consequently, low attrition rates. Taken together, this unique environment has consequently enabled the kind of data linkage across many registries and databases that we have performed with Population Data BC to facilitate a study of this nature and magnitude.

It is important to also highlight the limitations of the COAST Study. Importantly, our study did not have access to documented HIV testing data from the BC Centre for Disease Control (BC-CDC). However, as $95 \%$ of individuals who tested nominally within the $\mathrm{BC}-\mathrm{CDC}$ are also BC-CfE clients (BC Centre for Excellence in HIV/AIDS, Internal Data (2015)), we believe that we have captured most of the remaining $5 \%$ through the $\mathrm{BC}-\mathrm{MoH}$ records within Population Data BC through the aforementioned methods. We should note that although we believe we have captured the remaining $5 \%$, it would be useful in future updates to discern the validity of these cases by looking at laboratory markers such as CD4 cell count in addition to viral load. We are also limited in our ability to ascertain out-of-province events and to consistently identify Indigenous individuals who have been shown to be disproportionately affected by $\mathrm{HIV},{ }^{37}$ which will limit the type of analyses we can perform. Our assessment of specific HIV transmission groups, like people who use drugs and gay, bisexual and other men who have sex with men is limited by ascertainment of these groups by the BC-CfE. Additionally, administrative data can be prone to incomplete coding meaning that common limitations (eg, misclassification of exposures or outcomes) associated with the use of such data for research remains. The algorithm currently used in COAST to ascertain comorbidities associated with HIV is based on systems put in place by other studies and by a thorough review of these coding systems by a group of researchers associated with the study. Even though these deliberations are thorough, they are prone to misreporting and misclassifications of these exposures. In the future, we hope to validate many of these exposures in a series of small studies. Although loss to follow-up is a common concern in cohort studies, we were able to minimise loss through extensive data linkages across multiple data sources. Finally, since the absence of unmeasured confounding cannot be completely ruled out in observational studies such as this one, associations drawn from the study cannot be viewed as causal.

\section{CONCLUSION}

In summary, COAST is a robust population-based cohort study that was created within a fully subsidised health care setting and is ideally suited to investigate health outcomes and health care resource use among ageing HIV-positive individuals and the general population. COAST provides an important and unique avenue to better understand the association between cART uptake and health trends among PLHIV over time by employing a longitudinal comparison with the general population as a natural reference. Ongoing and future analyses using the COAST Study data will provide unique insights and empirical evidence to inform health policymakers' and HIV service providers' effort to better design programmes, devise and implement strategies and interventions to facilitate a more effective and efficient use of health care resources with the overall goal of improving population health outcomes. 
Author affiliations

${ }^{1}$ British Columbia Centre for Excellence in HIV/AIDS, St. Paul's Hospital, Vancouver, $\mathrm{BC}$, Canada

${ }^{2}$ Faculty of Health Sciences, Simon Fraser University, Burnaby, BC, Canada

${ }^{3}$ British Columbia Centre for Disease Control, Provincial Health Services Authority, Vancouver, BC, Canada

${ }^{4}$ Northern Ontario School of Medicine, Laurentian University, Sudbury, ON, Canada ${ }^{5}$ Department of Medicine, University of British Columbia, Vancouver, BC, Canada

${ }^{6}$ Centre for Clinical Epidemiology and Evaluation, Vancouver Coastal Health

Research Institute, Vancouver, BC, Canada

${ }^{7}$ Division of Cardiology, Providence Health Care, Vancouver, BC, Canada

Acknowledgements The authors thank the COAST study participants, BC-CfE, BC Cancer Agency, BC Ministry of Health, BC Vital Statistics Agency, PharmaNet and the institutional data stewards for granting access to the data, and Population Data BC, for facilitating the data linkage process. The authors also thank Monique Gagne, Brent Gali, James Nakagawa and other support staff at these institutions for administrative assistance with data access and preparation. The COAST steering committee includes: JSGM, RSH, OE, MWH, VDL and PS.

Contributors Study concept and design: OE, JSGM, RSH; acquisition of data: OE, MWH, HS, AC, PS, JSGM, RSH; drafting of the manuscript: OE; critical revision of the manuscript for important intellectual content and for final approval: $\mathrm{OE}, \mathrm{MWH}, \mathrm{KS}$, HS, AC, PS, VDL, BN, DGTW, SAL, JSGM, RSH; acquisition of funding: OE, AC, VDL, BN, DGTW, SAL, JSGM, RSH. All authors have read and approved the final version.

Funding COAST is funded by the Canadian Institutes of Health Research, through an Operating Grant (no: 130419), a Foundation Award to RSH (no: 143342) and support from the BC-CfE.

Disclaimer All inferences, opinions, and conclusions drawn in this manuscript are those of the authors, and do not reflect the opinions or policies of the data stewards or the funders.

Competing interests $\mathrm{OE}$ is supported by a Canadian Institutes of Health Research doctoral award. MWH has received grant support from the National Institute on Drug Abuse (NIDA R01DA031043-01) and has received honoraria for speaking engagements and/or consultancy meetings from the following: Bristol Myers Squibb, Gilead, Merck, Ortho-Janssen, Pfizer, Sunovion, Vertex Pharmaceuticals and Viiv. AC is supported by a CANOC Centre Scholar Award. VDL is funded by a grant from the Canadian Institutes of Health Research (PJT-148595), by a Scholar Award from the Michael Smith Foundation for Health Research and a New Investigator award from the Canadian Institutes of Health Research. JSGM is supported with grants paid to his institution by the British Columbia Ministry of Health and by the US National Institutes of Health (R01DA036307). He has also received limited unrestricted funding, paid to his institution, from Abbvie, Bristol-Myers Squibb, Gilead Sciences, Janssen, Merck and ViiV Healthcare. RSH has held grant funding in the last 10 years from the National Institutes of Health, Canadian Institutes of Health Research, Health Canada, Merck, and the Social Sciences and Humanities Research Council of Canada. The remaining authors have no disclosures to declare.

Ethics approval Ethical approval for this study was received from the University of British Columbia/ Providence Health Care Research Ethics Board (\#H09-02905) and Simon Fraser University Office of Research Ethics (\#2013s0566). The study complies with the BC Freedom of Information and Protection of Privacy Act (FIPPA) and did not require informed consent as it is conducted retrospectively for research and statistical purposes only using anonymised data.

Provenance and peer review Not commissioned; externally peer reviewed.

Data sharing statement The COAST team is open to potential research collaborations with other scientists, with the proviso that analysis of the data is currently authorised to occur at BC-CfE only, owing to ethical considerations in relation to relevant privacy legislation and research agreement. Researchers interested in collaboration should contact the Principal Investigator, RSH, at rhogg@ sfu.ca with their expression of interest.

Author note In terms of collaboration, the COAST team welcomes input from external investigators regarding research proposals or opportunities for collaboration to pursue joint or comparative analyses. All proposals are reviewed by the COAST Steering Committee for approval. For additional information, please contact the Principal Investigator, RSH, at: rhogg@sfu.ca.

Open Access This is an Open Access article distributed in accordance with the Creative Commons Attribution Non Commercial (CC BY-NC 4.0) license, which permits others to distribute, remix, adapt, build upon this work non-commercially, and license their derivative works on different terms, provided the original work is properly cited and the use is non-commercial. See: http://creativecommons.org/ licenses/by-nc/4.0/

(C) Article author(s) (or their employer(s) unless otherwise stated in the text of the article) 2018. All rights reserved. No commercial use is permitted unless otherwise expressly granted.

\section{REFERENCES}

1. Hogg RS, Heath KV, Yip B, et al. Improved survival among HIVinfected individuals following initiation of antiretroviral therapy. JAMA 1998;279:450-4

2. Palella FJ, Delaney KM, Moorman AC, et al. Declining morbidity and mortality among patients with advanced human immunodeficiency virus infection. HIV Outpatient Study Investigators. N Engl J Med 1998:338:853-60.

3. Mocroft A, Ledergerber B, Katlama C, et al. Decline in the AIDS and death rates in the EuroSIDA study: an observational study. Lancet 2003:362:22-9.

4. Samji H, Cescon A, Hogg RS, et al. Closing the gap: increases in life expectancy among treated HIV-positive individuals in the United States and Canada. PLoS One 2013;8:e81355.

5. World Health Organization (WHO). HIV/AIDS in Europe - Moving from death sentence to chronic disease management. 2006. http:// www.euro.who.int/en/what-we-publish/abstracts/hivaids-in-europe.moving-from-death-sentence-to-chronic-disease-management (accessed 16 Jul 2017).

6. Luther VP, Wilkin AM. HIV infection in older adults. Clin Geriatr Med 2007;23:567-83.

7. Effros RB, Fletcher CV, Gebo K, et al. Aging and infectious diseases: workshop on HIV infection and aging: what is known and future research directions. Clin Infect Dis 2008;47:542-53.

8. Rodriguez-Penney AT, ludicello JE, Riggs PK, et al. Co-morbidities in persons infected with HIV: increased burden with older age and negative effects on health-related quality of life. AIDS Patient Care STDS 2013;27:5-16.

9. Goulet JL, Fultz SL, Rimland D, et al. Aging and infectious diseases: do patterns of comorbidity vary by HIV status, age, and HIV severity? Clin Infect Dis 2007;45:1593-601.

10. Smit M, Brinkman K, Geerlings S, et al. Future challenges for clinical care of an ageing population infected with HIV: a modelling study. Lancet Infect Dis 2015;15:810-8.

11. BC Centre for Excellence in HIV/AIDS. Drug treatment program and laboratory. 2014. http://www.cfenet.ubc.ca/drug-treatment-program

12. Population Data BC. About population data BC. 2017. http://www. popdata.bc.ca/aboutus (accessed 6 Jul 2017).

13. British Columbia Ministry of Health (2014). Medical Services Plan (MSP) Payment Information File. Population Data BC. Data Extract. MOH. 2014.http://www.popdata.bc.ca/data

14. British Columbia Ministry of Health (2014). Consolidation file (MSP Registration and Premium Billing). Population Data BC. Data Extract. MOH. 2014.http://www.popdata.bc.ca/data

15. Canadian Institute for Health Information (2014). Discharge Abstract Database (Hospital Separations). Population Data BC. Data Extract. $\mathrm{MOH}$. 2014.http://www.popdata.bc.ca/data

16. British Columbia Ministry of Health (2012). Mental Health. Population Data BC. Data Extract. MOH. 2014.http://www.popdata.bc.ca/data

17. BC Cancer Agency Registry Data (2014). Population Data BC. Data Extract. BC Cancer Agency 2014.http://www.popdata.bc.ca/data

18. British Columbia Vital Statistics Agency (2014). Vital Statistics Deaths. Population Data BC. Data Extract. BC Vital Statistics Agency. 2014.http://www.popdata.bc.ca/data

19. British Columbia Ministry of Health (2014). PharmaCare. Population Data BC. Data Extract. MOH. 2014.http://www.popdata.bc.ca/data

20. British Columbia Ministry of Health (2014). PharmaNet. BC Ministry of Health. Data Extract. Data Stewardship Committee. 2014. http:// www.popdata.bc.ca/data.

21. Antoniou T, Zagorski B, Loutfy MR, et al. Validation of case-finding algorithms derived from administrative data for identifying adults living with human immunodeficiency virus infection. PLoS One 2011;6:e21748.

22. Nosyk B, Colley G, Yip B, et al. Application and validation of case-finding algorithms for identifying individuals with human immunodeficiency virus from administrative data in British Columbia, Canada. PLoS One 2013;8:e54416.

23. British Columbia Centre for Disease Control. HIV in British Columbia: Annual Surveillance Report 2014. 2015 http://www.bccdc.ca/healthprofessionals/data-reports/annual-surveillance-reports accessed 16 Jul 2017. 
24. Public Health Agency of Canada (PHAC). HIV and AIDS in Canada: Surveillance Report to December 31, 2014. 2015 https://www. canada.ca/en/public-health/services/publications/diseasesconditions/hiv-aids-canada-surveillance-report-december-31-2014. html (accessed 16 Jul 2017).

25. Population Data BC. Data linkage. https://www.popdata.bc.ca/ datalinkage (accessed 6 Jul 2017)

26. Patterson S, Cescon A, Samji H, et al. Cohort profile: HAART Observational Medical Evaluation and Research (HOMER) Cohort. Int J Epidemiol 2015;44:58-67.

27. Heath K, Samji H, Nosyk B, et al. Cohort profile: seek and treat for the optimal prevention of HIV/AIDS in British Columbia (STOP HIV/ AIDS BC). Int J Epidemiol 2014;43:1073-81.

28. May MT, Ingle SM, Costagliola D, et al. Cohort profile: Antiretroviral Therapy Cohort Collaboration (ART-CC). Int J Epidemiol 2014;43:691-702.

29. Hogg RS, Eyawo O, Collins AB, et al. Health-adjusted life expectancy in HIV-positive and HIV-negative men and women in British Columbia, Canada: a population-based observational cohort study. Lancet HIV 2017;4:e270-6.

30. Samji $\mathrm{H}$, Zhang W, Eyawo $\mathrm{O}$, et al. Rates and predictors of injury in a population-based cohort of people living with HIV. AIDS 2017;31:295-304.
31. Eyawo O, Franco-Villalobos C, Hull MW, et al. Changes in mortality rates and causes of death in a population-based cohort of persons living with and without HIV from 1996 to 2012. BMC Infect Dis 2017:17:174

32. Closson K, Osborne C, Smith DM, et al. Factors associated with mood disorder diagnosis among a population based Cohort of men and women living with and without HIV in British Columbia between 1998 and 2012. AIDS Behav 2017.

33. Justice AC, Landefeld CS, Asch SM, et al. Justification for a new cohort study of people aging with and without HIV infection. J Clin Epidemiol 2001;54:S3-8.

34. Patel P, Hanson DL, Sullivan PS, et al. Incidence of types of cancer among HIV-infected persons compared with the general population in the United States, 1992-2003. Ann Intern Med 2008;148:728-36.

35. Dechartres A, Boutron I, Nizard R, et al. Knee arthroplasty: disabilities in comparison to the general population and to hip arthroplasty using a French national longitudinal survey. PLoS One 2008;3:e2561.

36. Yu AP, Tang J, Xie J, et al. Economic burden of psoriasis compared to the general population and stratified by disease severity. Curr Med Res Opin 2009;25:2429-38.

37. Duncan KC, Reading C, Borwein AM, et al. HIV incidence and prevalence among aboriginal peoples in Canada. AIDS Behav 2011;15:214-27. 